\title{
Enhancement of larval immune system traits as a correlated response to selection for rapid development in Drosophila melanogaster
}

\author{
Punyatirtha Dey ${ }^{1}$, Kanika Mendiratta ${ }^{2}$, Joy Bose and Amitabh Joshi ${ }^{*}$ \\ Evolutionary Biology Laboratory, Evolutionary \& Organismal Biology Unit, Jawaharlal Nehru \\ Centre for Advanced Scientific Research, Jakkur P.O., Bengaluru 560 064, India.
}

1. Present address: Thermo Fisher Scientific, First Technology Place, 3EPIP, Whitefield, Bengaluru 560 066, India.

2. Present address: Aditi Staffing, Aditi Staffing Pvt Ltd, C4,Elm Building, Wing A Manyata SEZ

Business Park, Nagavara, Bengaluru 560 045, India.

E-mail addresses:

punyatirtha@gmail.com

kanika210488@gmail.com

caspase.joy@gmail.com

ajoshi@jncasr.ac.in

*: corresponding author

Running title: Larval immunity and development time

Key words: developmental rate; hemocytes; phenol oxidase; life-history tradeoff.

Main text: 2686 words; References: 29; Tables: 1; Figures: nil 


\section{Introduction}

We have shown earlier that the evolution of rapid development is accompanied by a correlated decrease in larval feeding rate and competitive ability in laboratory populations of Drosophila melanogaster (Prasad et al. 2001; Shakarad et al. 2005). Here, we show that our faster developing populations have evolved higher hemocyte density and phenol oxidase activity in the larval hemolymph. The increased hemocyte density could be responsible for the evolution of decreased feeding rate as hemocytes and the cephalopharyngeal musculature share common embryonic precursor cells (Kraajiveld et al. 2001). We also show that the bacterial load in larval food vials of the faster developing populations is substantially higher than in controls. Our results suggest that the evolution of reduced competitive ability in the faster developing populations is probably due to larval feeding rate trading off with enhanced larval immune system function. Enhanced larval immune function, in turn, is most likely selected for due to the role of hemocytes (Lanot et al. 2001, Wood and Jacinto 2007) and phenol oxidase (Pentz et al. 1986) in development, and perhaps also due to inadvertent selection on immune performance resulting from the higher bacterial load faced by larvae in the faster developing populations.

In nature, Drosophila larvae typically inhabit ephemeral habitats like rotten fruits, often at high density (Atkinson and Shorrocks 1997), conditions that are believed to favour the evolution of rapid pre-adult development (Clarke et al. 1961; Partridge and Fowler 1992). However, rapid development is also correlated with reduced adult body size (Chippindale et al. 1997; Nunney 1996), which is itself a strong correlate of reproductive fitness. Development time, thus, is a key life-history trait linking fitness 
components in the pre-adult and adult life-stages, and the various tradeoffs surrounding development time are believed to mediate the evolution of developmental and growth rates and body size in Drosophila. Consequently, direct and correlated responses to selection for rapid pre-adult development have been extensively studied in $D$. melanogaster over the last two decades or so, yielding important insights into the fitness consequences of genetic constraints that shape the evolution of development time (reviewed by Prasad and Joshi 2003).

For example, two sets of populations of D. melanogaster selected for rapid development in our laboratory independently evolved reduced larval feeding (cephalopharyngeal sclerite retraction) rate (Prasad et al. 2001; Rajamani et al. 2006). Most likely due in part to the reduced feeding rate, larvae from the faster developing populations were poorer competitors at high larval rearing density than their ancestral control counterparts (Joshi et al. 2001; Shakarad et al. 2005). This tradeoff between developmental rate and feeding rate indicates possible competition between these two traits for some common underlying resources. However, nothing is presently known about the nature of the limiting resources that affect the expression of both feeding rate and development rate, although studies on parasitoid-resistant populations of $D$. melanogaster suggest a possible explanation.

The evolution of increased parasitoid resistance in D. melanogaster under laboratory selection is accompanied by the correlated evolution of lower competitive ability due to a reduced larval feeding rate (Fellows et al. 1999; Kraajiveld et al. 1997), and an increased hemocyte density in the larval hemolymph (Kraajiveld et al. 2001). Upon parasitoid attack, larvae from these parasitoid resistant populations mount a more 
vigorous hemocyte-mediated encapsulation response compared to relatively susceptible controls (Fellows et al. 1999; Kraajiveld et al. 1997, 2001). Since both the hemocytes and the cephalopharyngeal musculature of larvae originate in the head mesoderm of the embryo, it appears that the parasitoid resistant larvae invest limiting cellular resources into hemocytes rather than cephalopharyngeal musculature during development and, consequently, have a reduced larval feeding rate that ultimately translates into reduced competitive ability (Kraajiveld et al. 2001).

While hemocytes are known to be important for defense against pathogens/parasitoid eggs in Drosophila (Lemaitre 2007), they are also essential for proper development (Lanot et al. 2001, Wood and Jacinto 2007). During metamorphosis, the hemocytes engulf the cells marked for destruction, thus playing a major role in tissue remodeling (Lanot et al. 2001). Hemocyte-deficient mutants of Drosophila do not complete metamorphosis and die as prepupae (Braun et al. 1998). Given the link between higher hemocyte density and decreased larval feeding rate (Kraajiveld et al. 2001) on the one hand, and hemocytes and development on the other, we hypothesized that our faster developing populations may have evolved a higher hemocyte density to be able to support metamorphosis in a substantially reduced pupal duration, about three-fourths that of controls (Modak 2009). The necessity of rapid development in these populations could therefore be responsible for the correlated evolution of reduced larval feeding rate.

The hemolymph of Drosophila larvae contains three types of hemocytes, namely plasmatocytes, crystal cells and lamellocytes (Braun et al. 1999). Plasmatocytes act as phagocytes, while crystal cells store phenol oxidase and other enzymes required for the melanization response (Braun et al. 1999, Lemaitre and Hoffman 2007). Lamellocytes are 
normally absent from hemolymph, and differentiate only on infection by parasitoids (Lemaitre and Hoffman 2007). In this study, we estimated the hemocyte density and phenol oxidase activity in larvae of our fast developing populations of $D$. melanogaster. We also investigated whether there was any difference between faster developing populations and controls in the bacterial load in the larval food medium of their respective culture vials.

\section{Materials and methods}

\section{Experimental populations}

We studied eight populations of D. melanogaster, of which four were ancestral controls $\left(\mathrm{JB}_{1-4}\right)$ and four had been subjected to selection for rapid development and early reproduction $\left(\mathrm{FEJ}_{1-4}\right)$ for about 350 generations at the time of this study. These populations and their maintenance regimes have been described in detail earlier (Prasad et al. 2000, 2001). Briefly, each FEJ population was derived from a single JB population: $\mathrm{FEJ}_{i}$ derived from $\mathrm{JB}_{i}, i=1-4$. All populations were maintained on a discrete generation cycle, at $25 \pm 1{ }^{\circ} \mathrm{C}$ and constant light and high humidity, on banana-jaggery food medium. The number of breeding adults was about 1800 in the JBs and about 1200 in the FEJs. The adult flies were maintained in Plexiglas cages $\left(25 \times 20 \times 15 \mathrm{~cm}^{3}\right)$, and the larvae in 8-dram glass vials $(9 \mathrm{~cm}$ height $\times 2.4 \mathrm{~cm}$ diameter $)$. The JB controls were maintained on a 21 day discrete cycle, with the eclosed adults being transferred to fresh vials on the $12^{\text {th }}$, $14^{\text {th }}$ and $16^{\text {th }}$ day after egg collection. Twelve days are sufficient for all viable individuals to eclose at the rearing larval density of $60-80$ eggs per $6 \mathrm{~mL}$ of food per vial used. On the $18^{\text {th }}$ day after egg collection, the flies were collected into a Plexiglas cage containing a Petri dish of food covered with yeast-acetic acid paste. The eggs laid by these flies on 
the $21^{\text {st }}$ day were collected off a fresh Petri dish with food and transferred to vials. The maintenance regime of the FEJs differed from the JBs in two important aspects, together imposing selection for rapid development and early reproduction relative to the controls. First, only the earliest 25 percent or so of the flies that eclosed in each food vial were collected into cages to form the breeding adult population. Second, the eggs from these adult flies were collected 3 days post-eclosion to initiate the next generation. At the time this study, the mean egg-to-adult development time for the FEJs was 7.1 days, compared to 9.3 days for the JBs (Modak 2009).

To eliminate any non-genetic parental effects of the different maintenance regimes, all eight JB and FEJ populations were maintained under identical control conditions for one generation prior to assays. Eggs collected from these "commongarden" flies were used to generate larvae, which were then used for the assays. Late $3^{\text {rd }}$ instar wandering stage larvae were used for all the assays.

\section{Measurement of hemocyte density in larval hemolymph}

Thin glass capillaries were pulled in flame to generate ultra-thin glass needles, which were then attached to $10 \mu \mathrm{L}$ pipette tips. Next, five larvae from a single population were placed on a slide, and their posterior ends were cut with scissors. The hemolymph from the five larvae was pooled on the slide. Looking through a stereomicroscope, $0.5 \mu \mathrm{L}$ of hemolymph was pipetted out, diluted with $0.05 \mathrm{M}$ phosphate buffer saline, $\mathrm{pH} 7.5$, and added to the counting chamber of a hemocytometer (Rohem Instruments, Nasik, India). The number of hemocytes was manually counted at 400X using an Eclipse E 200 microscope (Nikon, Guangdong, China). This procedure was carried out on larvae from 
all eight FEJ and JB populations, with five independent replicates of five pooled larvae each per population.

\section{Measurement of phenol oxidase activity per $\mu$ L of larval hemolymph}

As described above, 5-10 larvae were bled on a glass slide and $1 \mu \mathrm{L}$ of hemolymph was pipetted into $7 \mu \mathrm{L}$ of $10 \mathrm{mM}$ DOPA in $10 \mathrm{mM}$ PBS, pH 6.5 , and activated for 10 minutes at room temperature. After every 5 minutes, $1 \mu \mathrm{L}$ of reaction mixture was taken and the optical density at $470 \mathrm{~nm}$ was measured in a Nanodrop 1000 spectrophotometer (Thermo Scientific, Wilmington, USA). Change in optical density at $470 \mathrm{~nm}$ per hour was calculated from the linear region of the reaction, corrected for the dilution factor, and designated as phenol oxidase (PO) activity. For each population, five independent replicates were run and PO activity per $\mu \mathrm{L}$ of hemolymph was calculated for each replicate.

\section{Measurement of bacterial density in the larval food medium}

For each population, 70 eggs were placed on $6 \mathrm{~mL}$ of banana food in each of 10 replicate vials, and the larvae were allowed to develop for 3 days. After 3 days, $20 \mathrm{~mL}$ Luria broth (LB) was added to each vial and the food was lightly homogenized with a glass rod and suspended in LB. The suspension was centrifuged at $100 \mathrm{~g}$ for 5 minutes and the supernatant was diluted and plated on LB agar plates. The plates were incubated at $37^{\circ} \mathrm{C}$ for 18 hours, and colonies were counted manually to determine the bacterial density per mL of larval food for each vial.

\section{Statistical analyses}

The independent measurements of total hemocyte count, PO activity and bacterial density were taken as replicates within each population, and the data subjected to separate 
mixed model analyses of variance (ANOVA), with block, comprising of one FEJ population and the corresponding ancestral JB population, treated as a random factor crossed with selection regime. All analyses were implemented on Statistica $^{\mathrm{TM}}$ for Windows Release 5B (Statsoft Inc. 1995).

\section{Results}

\section{Hemocyte density in larval hemolymph}

The mean hemocyte density in the hemolymph of FEJ larvae was found to be 3.9 fold more than that in the ancestral control JB populations (Table 1), and the difference was significant $\left(F_{1,3}=78.028, p=0.0030\right)$.

\section{Phenol oxidase activity per unit volume of larval hemolymph}

The mean PO activity per $\mu \mathrm{L}$ hemolymph in FEJ larvae was found to be a 3.8 fold more than the ancestral control JB populations (Table 1), and the difference was significant $\left(F_{1,3}=282.492, p=0.0005\right)$.

\section{Bacterial load in larval food medium}

The amount of bacteria present in the larval food vials of the FEJ populations was found to be 5.8 fold more than that in the ancestral control JB populations (Table 1), and this difference was significant $\left(F_{1,3}=47.49, p=0.0063\right)$

\section{Discussion}

Our observation that the faster developing FEJ populations, earlier seen to have evolved decreased larval feeding rate (Prasad et al. 2001) and competitive ability (Shakarad et al. 2005), have also evolved a higher hemocyte density than ancestral controls (Table 1) is consistent with the tradeoff between hemocyte density and feeding rate of larvae from parasitoid resistant populations of D. melanogaster observed by Kraajiveld et al. (2001). 
Our finding thus supports the notion of competition for progenitor cells between investment in cephalopharyngeal musculature and hemocyte levels during early development (Kraajiveld et al. 2001). Accordingly, the correlated evolution of reduced larval feeding rate in the FEJ populations is likely to be driven at least in part by the necessity of increased hemocyte density for rapid development.

We also found that the FEJ larvae had increased PO activity per unit volume of hemolymph, compared to the ancestral JB controls (Table 1). PO activity in hemolymph is considered an immune parameter in insects (Lemaitre 2007) and is also related to the darkening of pupal and adult cuticle during development (Pentz et al. 1986). Overall, our finding that both hemocyte density and PO activity in the larval hemolymph increased in the FEJs also provides strong support for the notion of pleiotropy between developmental and immune functions in Drosophila larvae (Yixin et al. 2009). Yixin et al. (2009) selected populations of D. melanogaster for larval resistance to the pathogen Pseudomonas aeruginosa and observed the correlated evolution of reduced development time in the resistant populations. Our results suggest that the response of development time and larval immune system function to selection on one or the other trait is symmetric, a finding that strengthens the inference of pleiotropy between these traits.

Due to the dual role of hemocytes and PO in development and immunity, it is however difficult to infer unequivocally whether these traits in the FEJ populations evolved to facilitate faster development or in response to increased load of possibly nonpathogenic bacteria in the food medium, or both. A recent study on the insect Trichoplusia showed that even non-pathogenic, non-infectious bacteria present in the food of larvae can elicit an immune response and affect fitness components like pupation 
time (Freitak et al. 2007). In Drosophila cultures, microbial growth is suppressed by the feeding activity of larvae (Sang et al. 1949) and cultures with very few larvae per vial often get overgrown with bacteria and yeast compared to cultures with several tens of larvae per vial (A. Joshi, P. Dey, pers. obs.). Given that third instar FEJ larvae are smaller and slower feeders than their control counterparts but are maintained at the same density per vial (Prasad et al. 2001), it is possible that their inhibitory effect on bacterial growth in the larval food vials is less than that of the JB controls. The nature of this inhibitory effect of Drosophila larvae on microbial growth in the food vials is not clear. It could be that larvae check microbial population growth merely by feeding, or that larvae inhibit microbial growth through some hitherto unknown mechanism. Whatever the mechanism, however, it is clear that the FEJ culture vials harbor substantially larger bacterial populations at the third instar stage than control vials. In principle, this increased bacterial load could also directly select for enhanced immune system performance in the FEJ populations, independent of the effect of selection for rapid development, mediated through the multi-tasking role of hemocytes and melanization. Such inadvertent selection pressures are not unusual in experimental evolution studies (Prasad and Joshi 2003).

We have earlier found that our FEJ populations suffer greater adult mortality than controls when grown in the presence of Escherichia coli (Modak et al. 2009). Unlike larval immune function, adult immune function is seen to trade off with development time in insects: populations of the yellow fever mosquito, Aedes aegypti, selected for rapid development showed reduced adult immunity (Koella and Boete 2002) whereas selection for higher adult immunity in the Indian meal moth, Plodia interpunctella, resulted in correlated evolution of longer development time (Boots and Begon 1993). In 
general, it appears that larval and adult immunity in Drosophila have the potential to be correlated or uncorrelated, depending on which measures of immune function are assayed (Petersen et al. 1999; Fellous and Lazzaro 2011). At this time, it is difficult to say whether our present results together with those of Modek et al. (2009) indicate a specific tradeoff between larval and adult immune function in our populations. As noted by Modak et al. (2009), it is possible that the increased mortality of the FEJ adults in the presence of $E$. coli is due to their mounting an equal or greater immune response than JB adults and then succumbing faster to the starving conditions of the assay vials as they have less lipid reserves to start with. What does seem clear, however, is that the FEJ larvae exhibit increased immune function, at least for some measures of larval immunity.

\section{Acknowledgments}

We thank N. Rajanna and M. Manjesh for assistance in the laboratory. PD was supported by a Research Associateship from the Jawaharlal Nehru Centre for Advanced Scientific Research (JNCASR). KM was supported by a fellowship through the Project Oriented Biology Education program of the JNCASR. The work was supported in part by funds from the Department of Science and Technology, Government of India. Preparation of the manuscript was supported in part by a J. C. Bose National fellowship from the Department of Science and Technology, Government of India, to AJ. 


\section{References}

Atkinson W. and Shorrocks B. 1977 Breeding site specificity in the domestic species of Drosophila. Oceologia 29, 223-232.

Boots M. and Begon M. 1993 Trade-offs with resistance to a granulosis virus in the Indian meal moth. Func. Ecol. 7, 528-534.

Braun A., Hoffmann J. A. and Meister M. 1998 Analysis of the Drosophila host defense in domino mutant larvae, which are devoid of hemocytes. Proc. Natl. Acad. Sci. USA 95, 14337-14342.

Chippindale A. K., Alipaz J. A., Chen H. W. and Rose M. R. 1997 Experimental evolution of accelerated development in Drosophila. 1. Developmental speed and larval survival. Evolution 51, 1536-1551.

Clarke J. M., Maynard Smith J. and Sondhi K. C. 1961 Asymmetrical response to selection for rate of development in Drosophila subobscura. Genet. Res. 2, 70-81.

Fellous S. and Lazzaro B. P. 2011 Potential for evolutionary coupling and decoupling of larval and adult immune gene expression. Mol. Ecol. 20, 1558-1567.

Fellowes M. D. E., Kraajiveld A. R. and Godfray H. C. J. 1999 Association between feeding rate and defence against parasitoids in Drosophila melanogaster. Evolution 53, 1302-1305.

Freitak F., Wheat, C. W. Hecker D. G. and Vogel H. 2007 Immune system responses and fitness cost associated with consumption of bacteria in larvae of Trichoplusia ni. BMC Biol. 5, 56.

Joshi A., Prasad N. G., and Shakarad M. $2001 K$-selection, $\alpha$-selection, effectiveness and tolerance in competition: density-dependent selection revisited. J. Genet. 80, 63-75. 
Koella J. B. and Boete C. 2002 A genetic correlation between age at pupation and melanization immune response of the yellow fever mosquito Aedes aegypti. Evolution 56, 1074-1079.

Kraajiveld A. R. and Godfray H. C. J. 1997 Trade-off between parasitoid resistance and larval competitive ability in Drosophila malanogaster. Nature 389, 278-280.

Kraajiveld A. R., Limentani E. C. and Godfray H. C. J. 2001 Basis of the trade-off between parasitoid resistance and larval competitive ability in Drosophila melanogaster. Proc. R. Soc. Lond. B 268, 259-261.

Lanot R., Zachary D., Holder F. and Meister M. 2001 Postembryonic hematopoiesis in Drosophila. Dev. Biol. 230, 243-257.

Lemaitre B. and Hoffmann J. 2007 The host defense of Drosophila melanogaster Annu. Rev. Immunol. 25, 697-714.

Modak S. G. 2009 Evolution of life-history traits, canalization and reproductive isolation in laboratory populations of Drosophila melanogaster selected for faster pre-adult development and early reproduction. Ph.D. thesis, Jawaharlal Nehru Centre for Advanced Scientific Research, Bengaluru, India.

Modak S. G., Satish K. M., Mohan J., Dey S., Raghavendra N., Shakarad M. and Joshi A. 2009 A possible tradeoff between developmental rate and pathogen resistance in Drosophila melanogaster. J. Genet. 88, 253-256.

Nunney L. 1996 The response to selection for fast larval development in Drosophila melanogaster and its effect on adult weight: an example of a fitness trade off. Evolution 50, 1193-1204. 
Partridge L. and Fowler K. 1992 Direct and correlated responses to selection on age at reproduction in Drosophila melanogaster. Evolution 46, 76-91.

Pentz E. S., Black B. C. and Wright T. R. F. 1986 A diphenoloxidase gene is part of a cluster of genes involved in catecholamine metabolism and sclerotization in Drosophila. I. Identification of the biochemical defect in Dox-A2 [1(2) $37 \mathrm{Bf}]$ mutants. Genetics 112, 23-841.

Petersen U. M., Kadalayil L., Rehorn K. P., Hoshizaki D. K., Reuter R. and Engstrom Y. 1999 Serpent regulates Drosophila immunity genes in the larval fat body through an essential GATA motif. EMBO J. 18, 4013-4022.

Prasad, N. G. and Joshi A. 2003 What have two decades of laboratory life-history evolution studies on Drosophila melanogaster taught us? J. Genet. 82, 45-76.

Prasad N. G., Shakarad M., Anitha D., Rajamani M. and Joshi A. 2001 Correlated responses to selection for faster development and early reproduction in Drosophila: the evolution of larval traits. Evolution 55, 1363-1372.

Prasad N. G., Shakarad M., Gohil V. M., Sheeba V., Rajamani M. and Joshi A. 2000 Evolution of reduced pre-adult viability and larval growth rate in laboratory populations of Drosophila melanogaster selected for shorter developmental time Genet. Res. 76, 249-259.

Rajamani M., Raghavendra N., Prasad N. G., Archana N., Joshi A. and Shakarad M. 2006 Reduced larval feeding rate is a strong evolutionary correlate of rapid development in Drosophila melanogaster. J. Genet. 85, 209-212. 
Sang J. H., McDonald J. M. and Gordon C. 1949 The ecological determinants of population growth in a Drosophila culture. VI. The total population count. Physiol. Zool. 22, 223-235.

Shakarad M., Prasad N. G., Gokhale K., Gadagkar V., Rajamani M. and Joshi A. 2005 Faster development does not lead to correlated evolution of greater pre-adult competitive ability in Drosophila melanogaster. Biol. Lett. 1, 91-94.

Statsoft Inc. 1995 Statistica Vol. I: General Conventions and Statistics I. Statsoft Inc., Tulsa, OK, USA.

Wood W. and Jacinto A. 2007 Drosophila melanogaster embryonic haemocytes: masters of multitasking. Nat. Rev. Mol. Cell Biol. 8, 542-551.

Yixin H. Y., Chenoweth S. F. and McGraw E. A. 2009 Effective but costly evolved mechanisms of defense against a virulent opportunistic pathogen in Drosophila melnanogaster. PLoS Pathogens 5, e1000385. 
Table 1. Mean ( \pm s.e.) immune system related traits and bacterial loads in control (JB) and rapidly developing (FEJ) populations. Standard errors reflect the variation among means of the four replicate populations within each selection regime.

\begin{tabular}{|l|l|l|l|}
\hline $\begin{array}{c}\text { Selection } \\
\text { Regime }\end{array}$ & Hemocyte density & PO activity & $\begin{array}{l}\text { Density of non pathogenic } \\
\text { bacteria in food }\end{array}$ \\
\cline { 2 - 4 } & $\begin{array}{l}\text { (Number of } \\
\text { hemocytes per } \mathrm{mm}^{3} \\
\text { larval hemolymph })\end{array}$ & $\begin{array}{l}\left(\Delta \mathrm{OD}_{470} \text { per } \mu \mathrm{L} \text { of }\right. \\
\text { hemolymph per hour })\end{array}$ & $\left(\right.$ Colony forming unit $\left.\times 10^{-3} / \mathrm{mL}\right)$ \\
\hline JB & $5794 \pm 511$ & $3.92 \pm 0.24$ & $6.0 \pm 1.9$ \\
FEJ & $22685 \pm 2052$ & $15.04 \pm 0.81$ & $35.6 \pm 4.2$ \\
\hline
\end{tabular}

